\title{
An Improved and Scalable Synthesis of Insensitive High Explosive
}

\section{4,10-Dinitro-2,6,8,12-tetraoxa-4,10-diazaisowurtzitane (TEX)}

Mukesh B. Deshmukh, Amulrao U. Borse, Pramod P. Mahulikar and Dipak S. Dalal*

School of Chemical Sciences, North Maharashtra University, Jalgaon - 425 001, India

*Corresponding Email: dsdalal2007@gmail.com

Tel: +91-2572257432; Fax: +91-2572258406 


\section{Spectral Data}

\section{1,4-diformyl-2,3,5,6-tetrahydroxypiperazine}

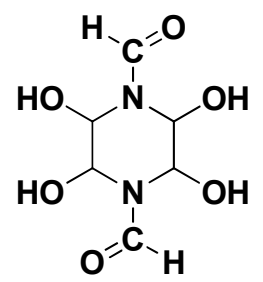

Molecular formula : $\mathrm{C}_{6} \mathrm{H}_{10} \mathrm{~N}_{2} \mathrm{O}_{6}$

Molecular weight $\quad: 206$

Mp $\quad: 224^{\circ} \mathrm{C}$

UV/VIS $\left(\lambda_{\max }\right.$, DMF) : No remarkable wavelength $(\lambda)$ maximum.

IR (KBr, $\left.v_{\max } / \mathbf{c m}^{-1}\right)$ : 3328 and $3226(\mathrm{OH}), 2711$ and $1667(\mathrm{CHO}), 1434$ and $1402\left(\mathrm{sp}^{3} \mathrm{C}-\mathrm{H}\right.$ bend), 1292( $\left.\mathrm{sp}^{2} \mathrm{C}-\mathrm{N}\right), 1071$ and $1050(\mathrm{C}-\mathrm{O}), 934,654$.

${ }^{1}$ H NMR (400 MHz, DMSO-d , Me $_{4}$ Si) $\delta_{\mathrm{H}}: 6.059\left(\mathrm{dd}, 2 \mathrm{H}, J_{\mathrm{d}, \mathrm{c}} 12, J_{\mathrm{d}, \mathrm{a}} 5 \mathrm{~Hz}, \mathrm{H}_{\mathrm{d}}\right), 5.925(\mathrm{dd}$, $\left.2 \mathrm{H}, J_{\mathrm{c}, \mathrm{d}} 12, J_{\mathrm{c}, \mathrm{b}} 6 \mathrm{~Hz}, \mathrm{H}_{\mathrm{c}}\right), 5.487\left(\mathrm{t}, 2 \mathrm{H}, J_{\mathrm{b}, \mathrm{c}} 6, J_{\mathrm{b}, \mathrm{d}} 12 \mathrm{~Hz}, \mathrm{H}_{\mathrm{b}}\right), 4.993\left(\mathrm{dd}, 2 \mathrm{H}, J_{\mathrm{a}, \mathrm{d}} 5, J_{\mathrm{a}, \mathrm{c}} 12 \mathrm{~Hz}\right.$ $\left.\mathrm{H}_{\mathrm{a}}\right), 8.263\left(\mathrm{~s}, 1 \mathrm{H}, \mathrm{H}_{\mathrm{e}}\right), 8.268\left(\mathrm{~s}, 1 \mathrm{H}, \mathrm{H}_{\mathrm{f}}\right) \mathrm{ppm}$.

${ }^{13}$ C NMR (100 MHz, DMSO-d $\left.\boldsymbol{d}_{6}+\mathbf{C D C l}_{3}, \mathbf{M e}_{4} \mathrm{Si}\right) \delta_{\mathrm{C}}:$ 72.33, 72.66, 164.33, $164.40 \mathrm{ppm}$. MS (m/z) : $204.9(\mathrm{M}-1)$ 


\section{4,10-dinitro-2,6,8,12-tetraoxa-4,10-diazaisowurtzitane}

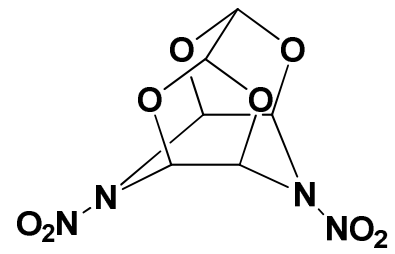

Molecular formula : $\mathrm{C}_{6} \mathrm{H}_{6} \mathrm{~N}_{4} \mathrm{O}_{8}$

Molecular weight $\quad: 262$

Мp

$$
: 305^{\circ} \mathrm{C}
$$

UV/VIS $\left(\lambda_{\max }\right.$, DMF $): 266 \mathrm{~nm}$

IR (KBr, $\left.v_{\max } / \mathbf{c m}^{-1}\right)$ : 3080-3020 (C-H), 1594 (N-O asym), 1326 (N-O sym), 1317, 1287 and $1252(\mathrm{~N}-\mathrm{C}), 1135$ and $1115(\mathrm{C}-\mathrm{O}), 898,875$.

${ }^{1}$ H NMR (400 MHz, DMSO-d ${ }_{6}$, Me $\left._{4} \mathbf{S i}\right) \delta_{\mathrm{H}}: 6.980\left(\mathrm{~s}, 4 \mathrm{H}, \mathrm{H}_{\mathrm{A}}\right), 5.933\left(\mathrm{~s}, 2 \mathrm{H}, \mathrm{H}_{\mathrm{B}}\right) \mathrm{ppm}$.

${ }^{13}$ C NMR (100 MHz, DMSO-d 6 + CDCl $\left._{3}, \mathbf{M e}_{4} \mathrm{Si}\right) \delta_{\mathrm{C}}: 82.48\left(\mathrm{C}_{1}\right), 102.56\left(\mathrm{C}_{2}\right) \mathrm{ppm}$. $\operatorname{MS}(\boldsymbol{m} / \boldsymbol{z}): 262(\mathrm{M}+), 218\left(\mathrm{M}-\mathrm{NO}_{2}\right)$. 


\section{Spectra}

FTIR, ${ }^{1} \mathrm{H}$ NMR, ${ }^{13} \mathrm{C}$ NMR and Mass spectra of synthesised compounds.

\begin{tabular}{ll}
\hline Sr. No. & Spectra \\
\hline 1 & FTIR spectrum of DFTHP \\
3 & ${ }^{1}$ H NMR spectrum of DFTHP \\
4 & ${ }^{13}$ C NMR spectrum of DFTHP \\
5 & Mass spectrum of DFTHP \\
6 & FTIR spectrum of TEX \\
7 & ${ }^{1}$ H NMR spectrum of TEX \\
8 & ${ }^{13}$ C NMR spectrum of TEX \\
9 & Mass spectrum of TEX \\
10 & HPLC of TEX \\
\hline
\end{tabular}




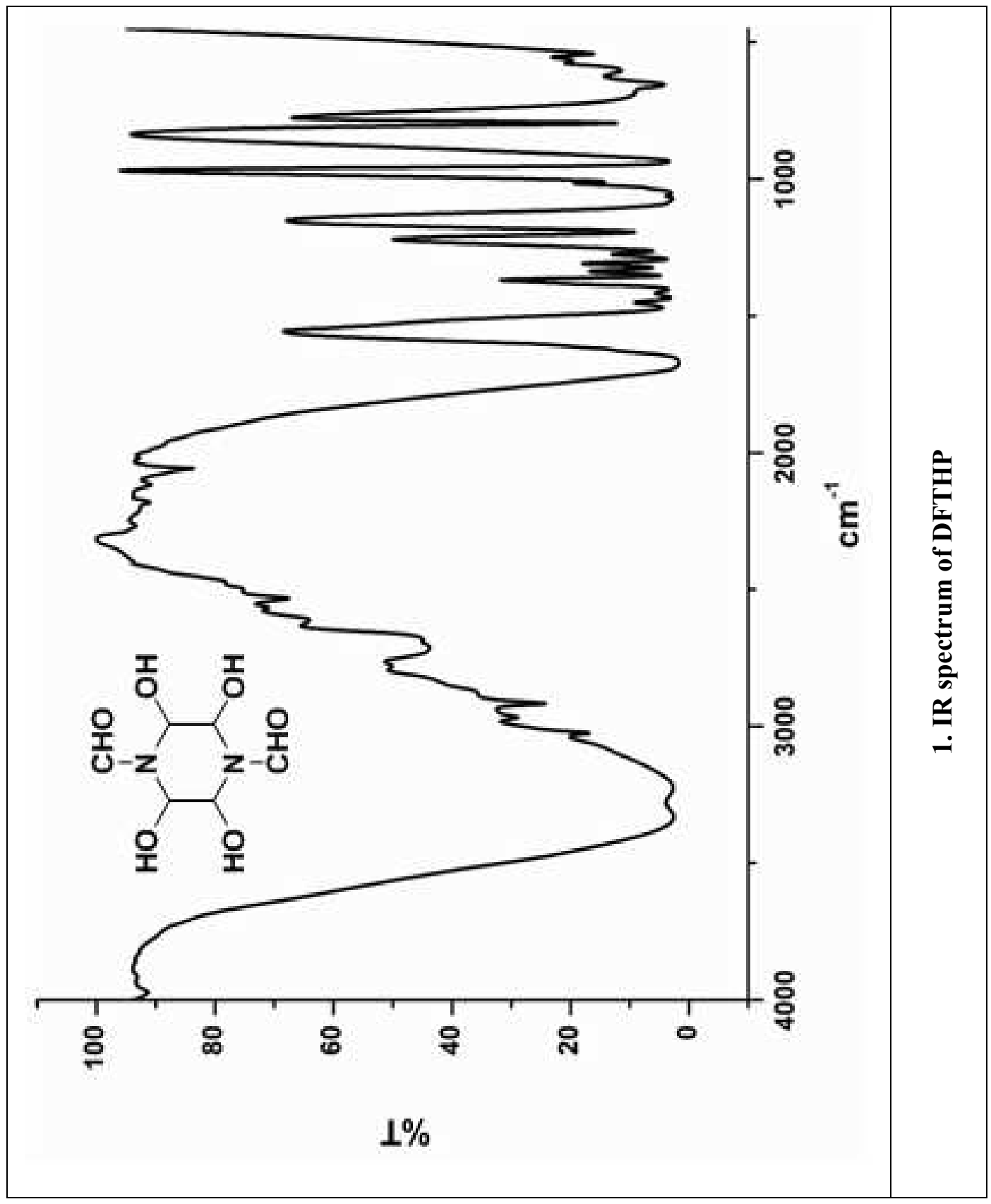




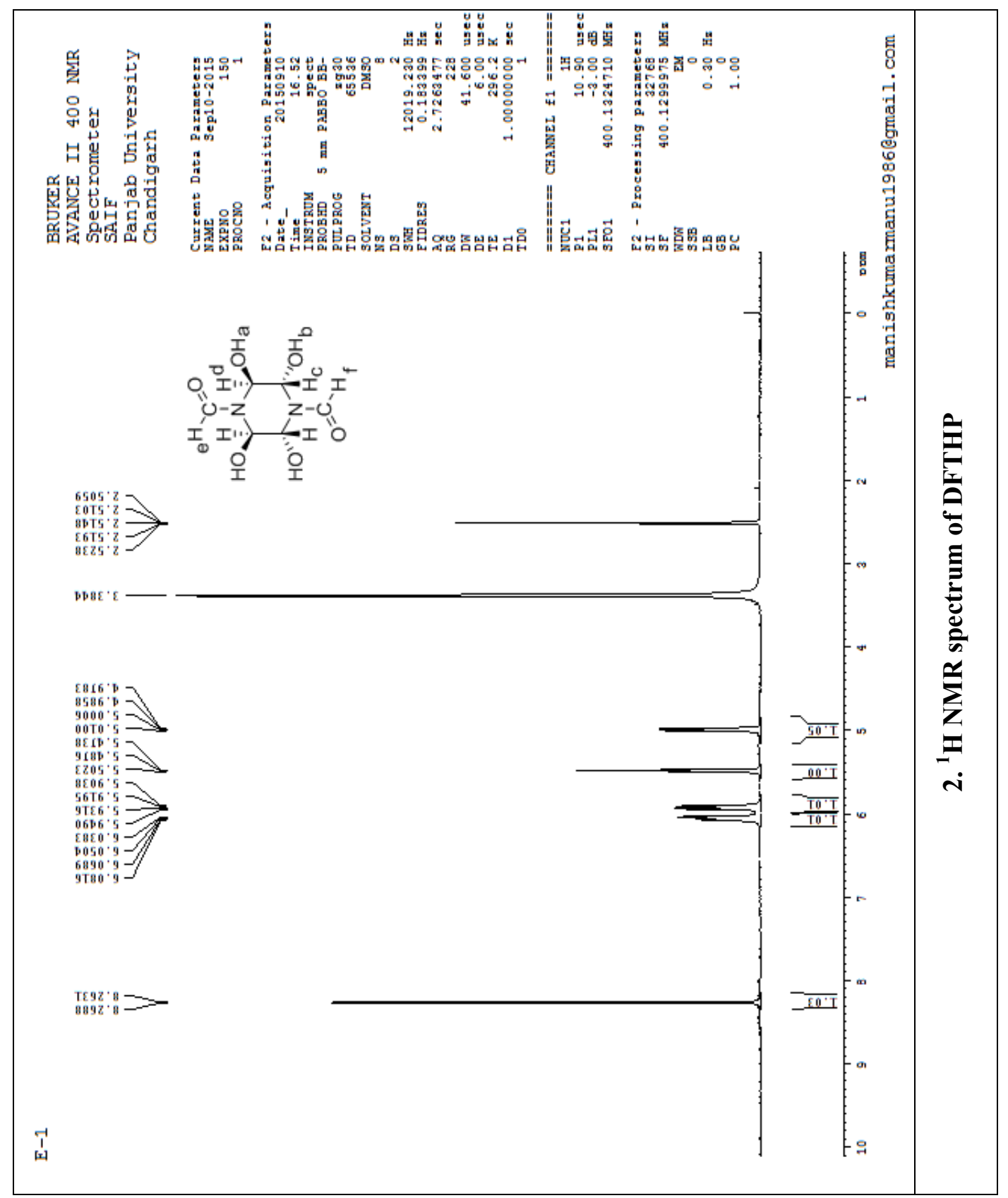




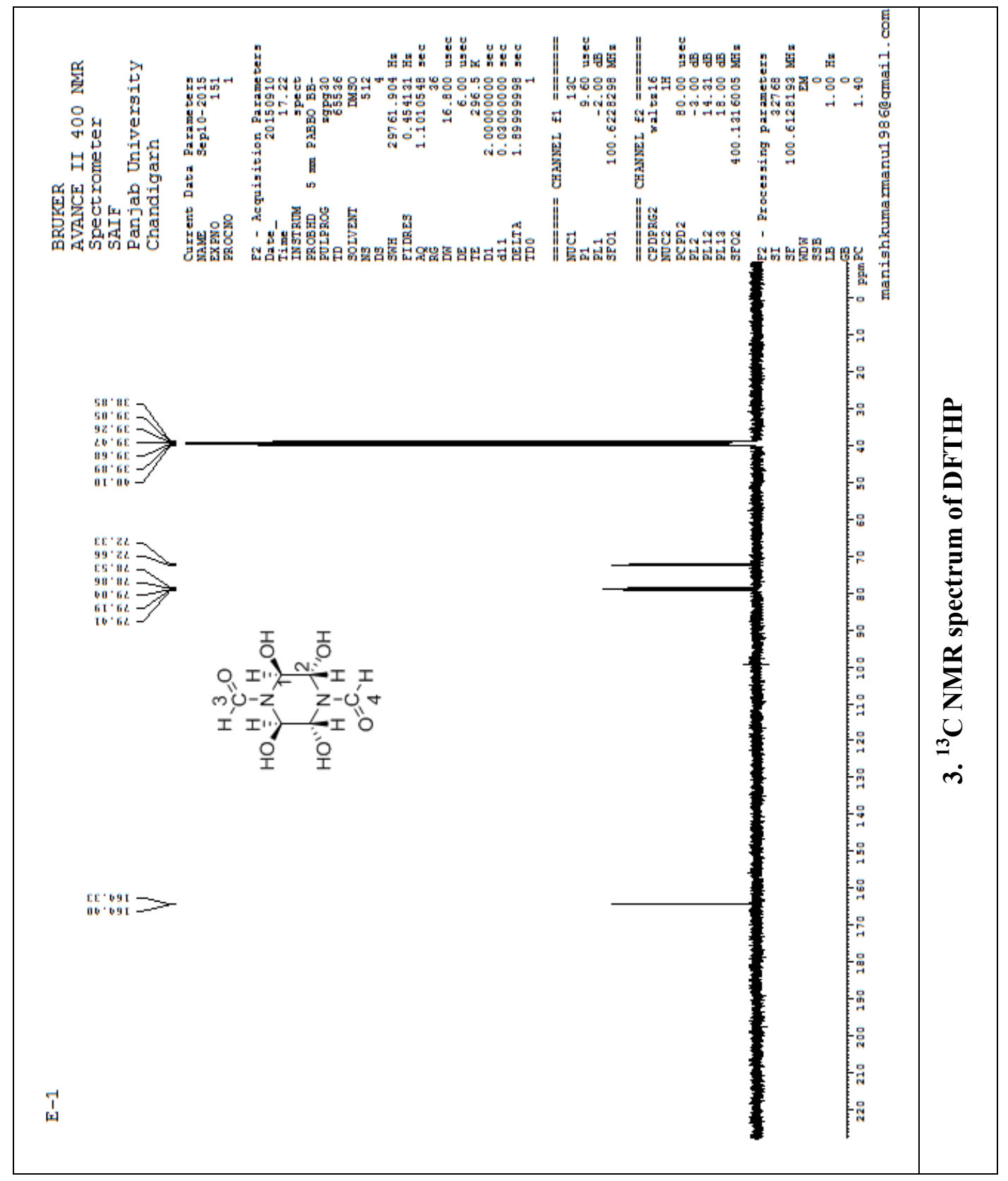




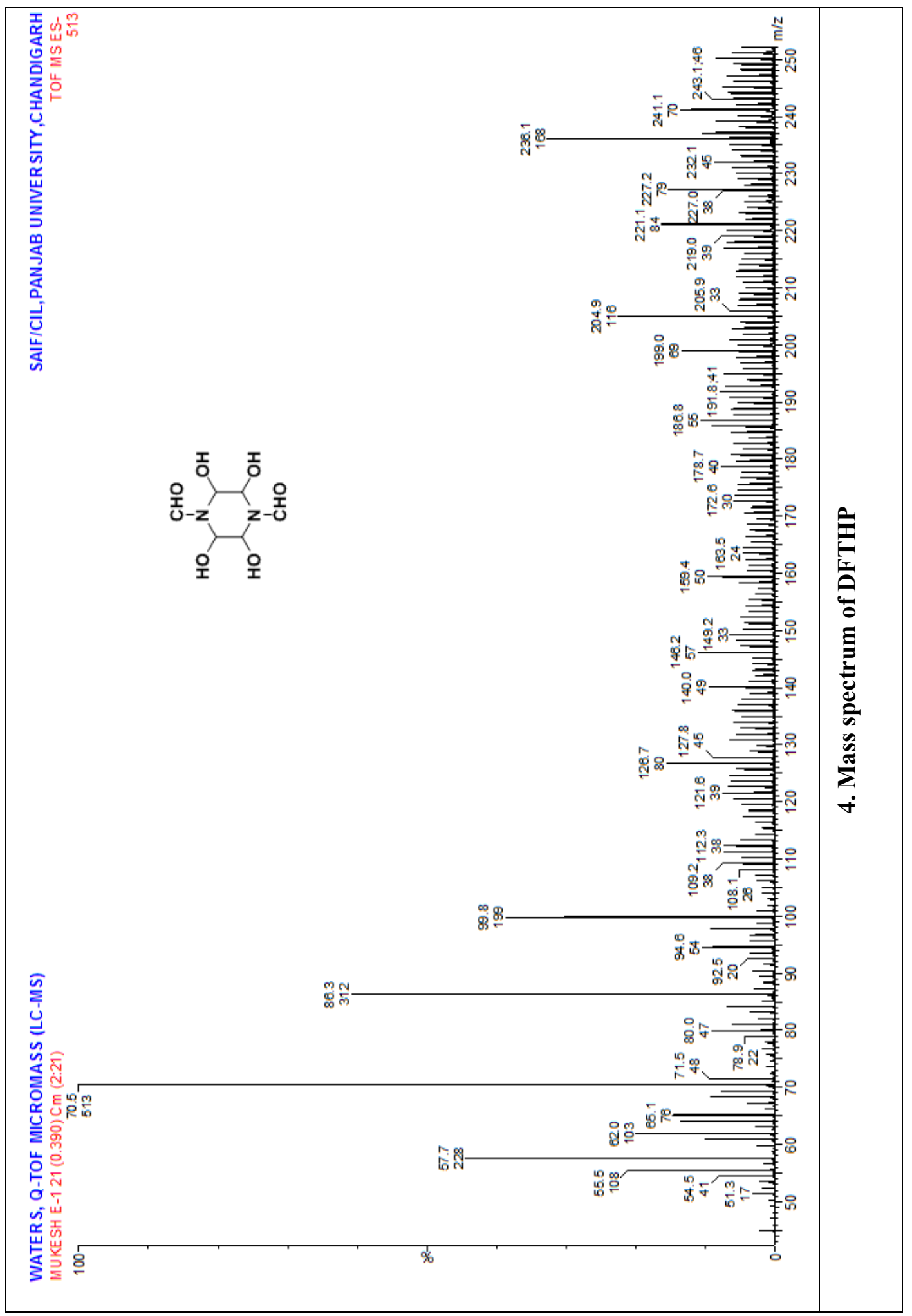




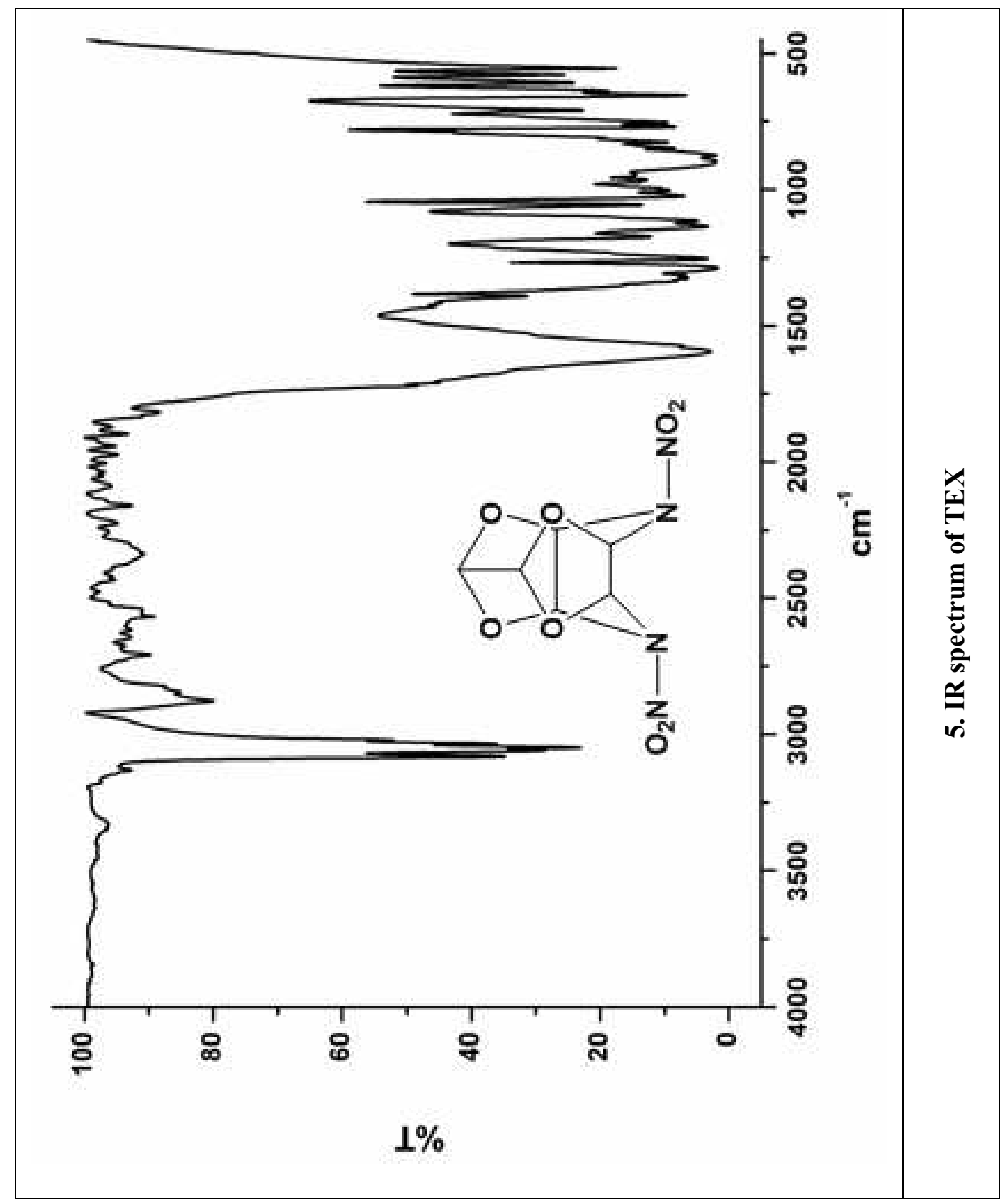




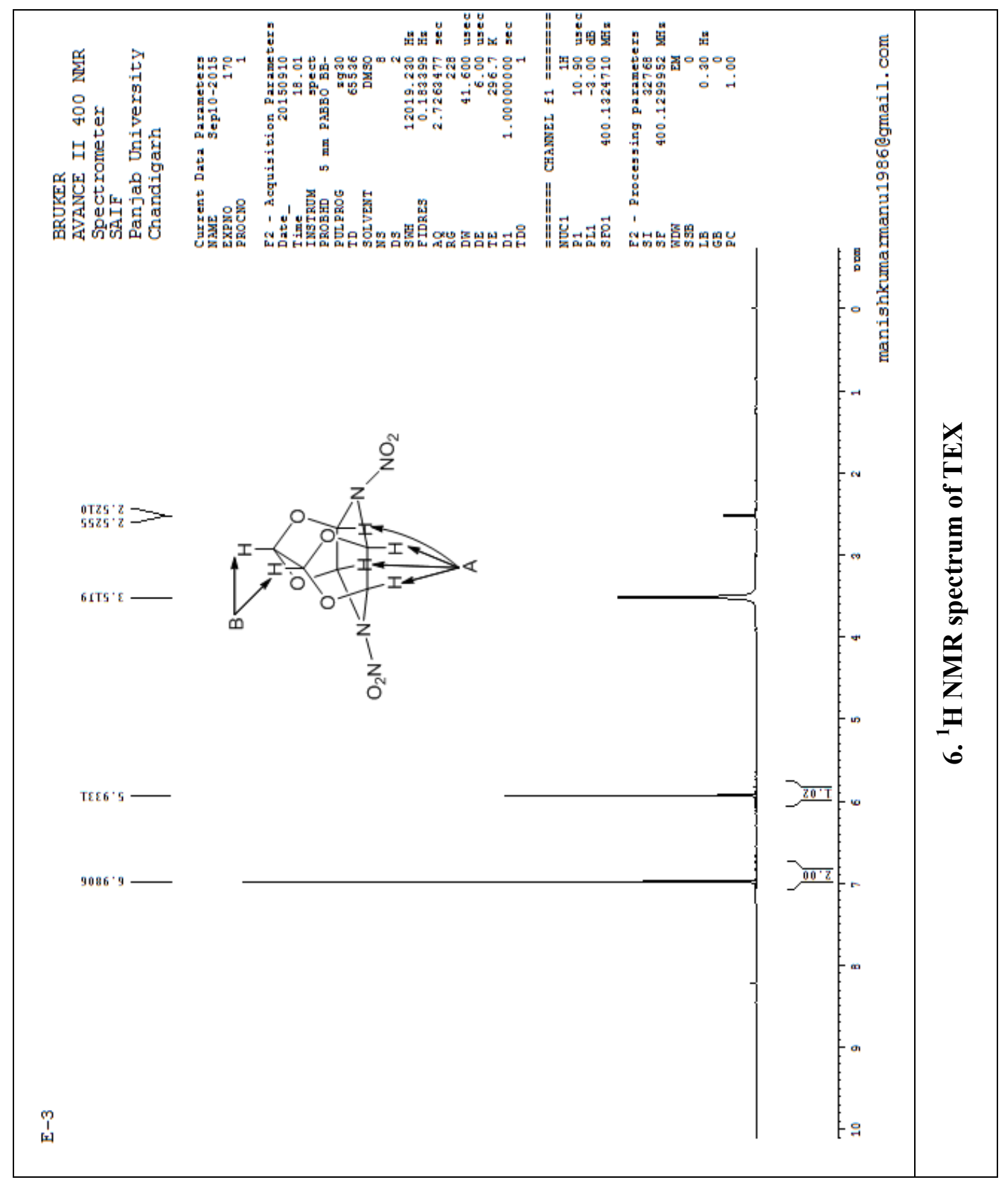




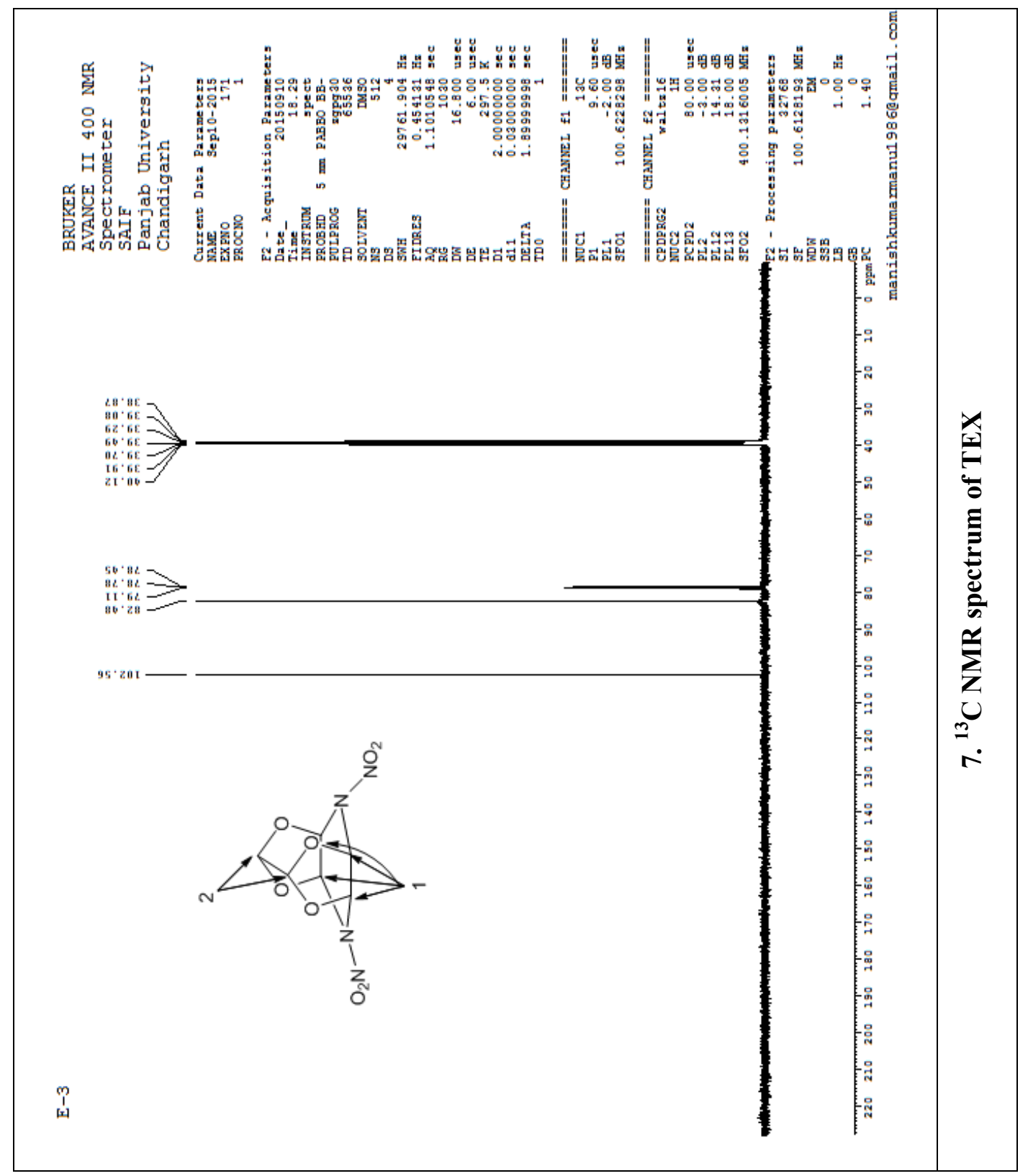




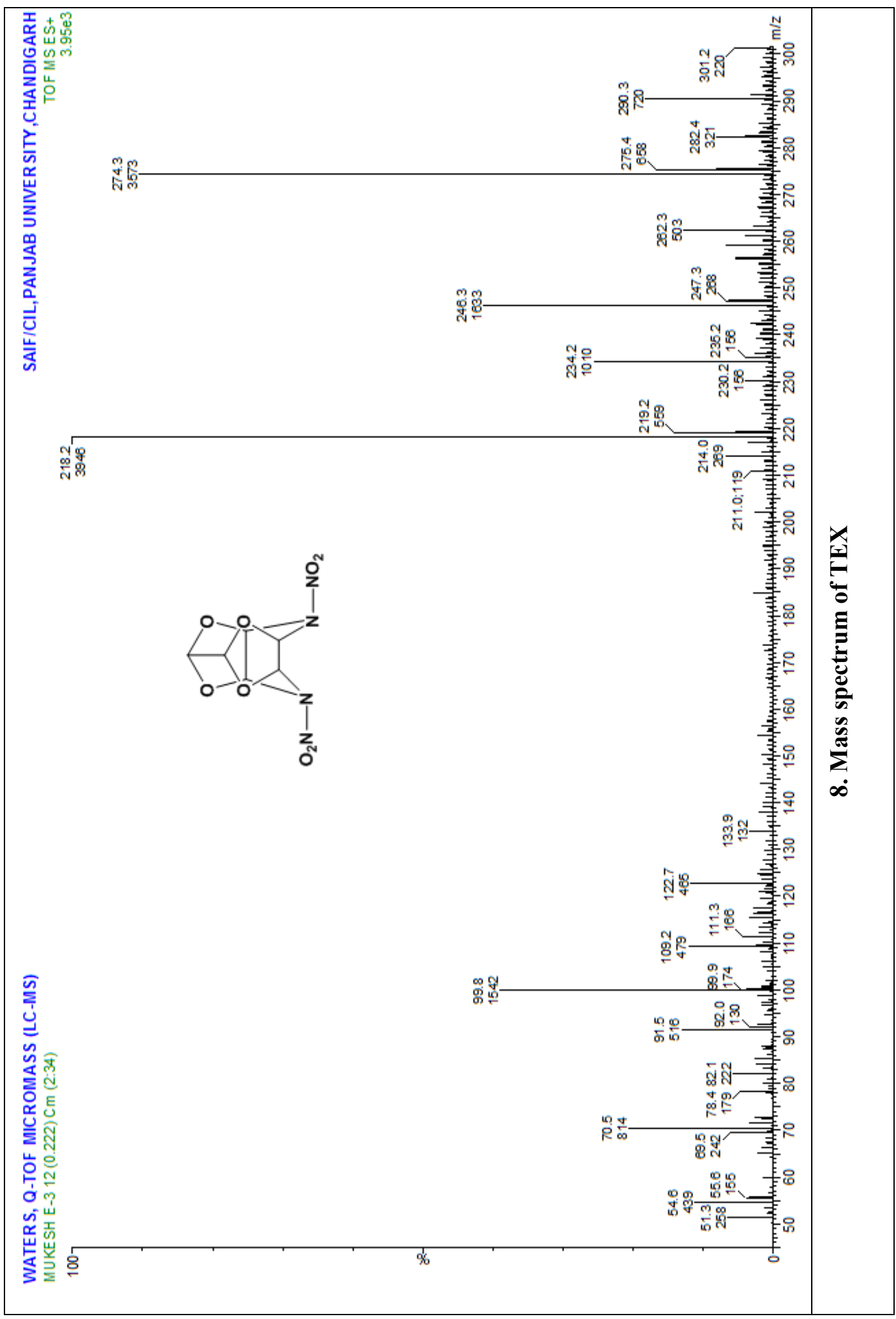




\section{HPLC Analysis Report}

Post: R.S.I.T.C, JALGAON 9( wuw.hplctrainingjal.com)

Name: ANIL VISPUTE

Analysis

Sample: 0052 E2 smp 1

File: 0188.RAW

Date: $\quad 2016-05-28$ AM 11:04:29

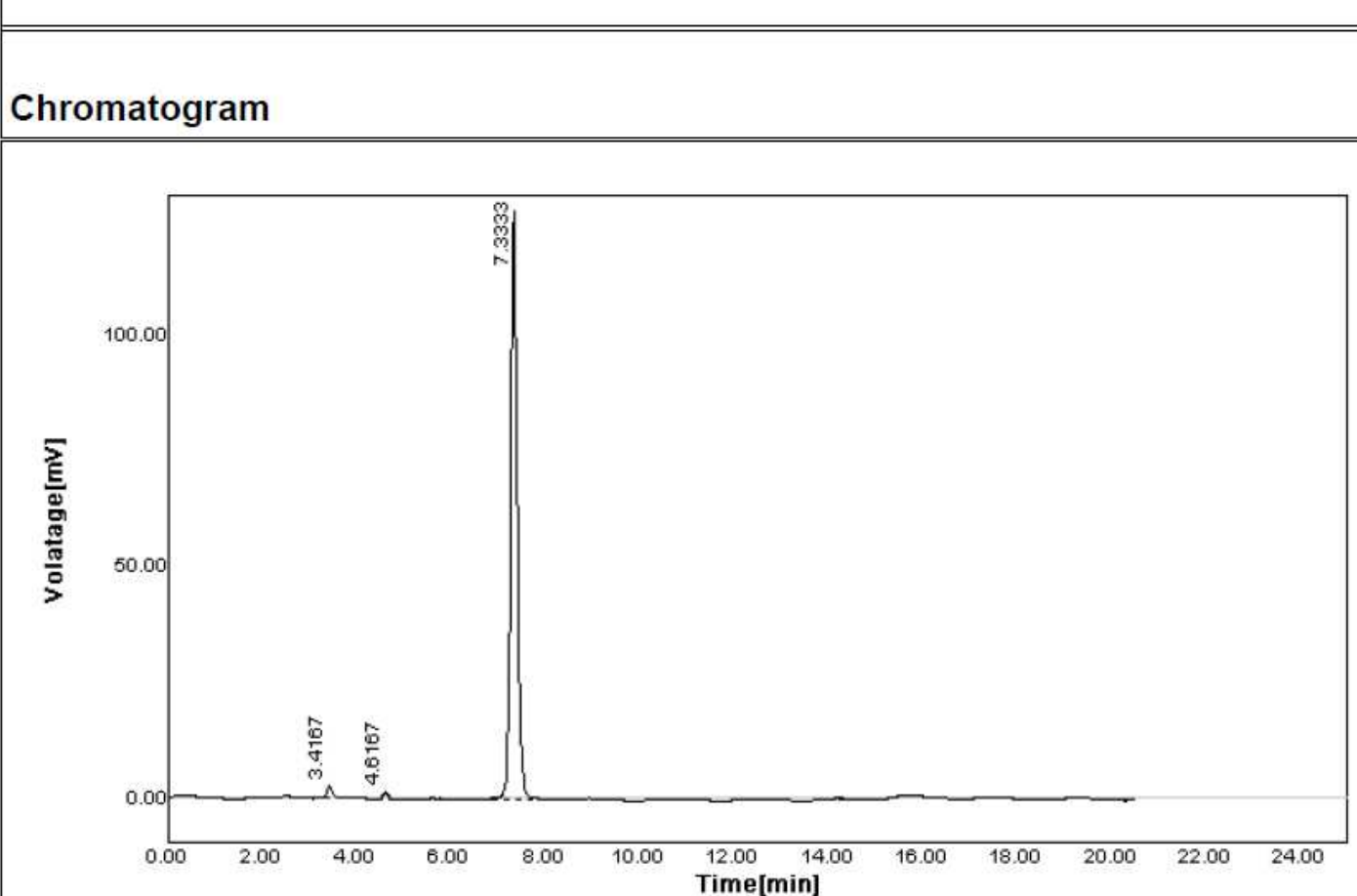

Result

\begin{tabular}{|c|c|c|c|c|c|c|c|}
\hline No. & RT[min] & Name & Area $\left[\mathrm{mV}^{*} \mathrm{~s}\right]$ & Area $\%$ & TP & TF & Resolution \\
\hline 1 & 3.4167 & & 16.2469 & 1.30 & 4755.9 & 1.3000 & 0.0000 \\
\hline 2 & 4.6167 & & 12.6748 & 1.02 & 6648.1 & 1.1875 & 4.8000 \\
\hline 3 & 7.3333 & & 1216.9492 & 97.68 & 13253.7 & 1.1000 & 9.5882 \\
\hline Sum & & & 1245.8710 & & & & \\
\hline
\end{tabular}




\section{HPLC Analysis Report}

Post: R.S.I.T.C, JALGAON 9 ( www.hplctrainingjal.com)

Name: ANIL VISPUTE

\section{Analysis}

Sample: 0053 E3 smp

File: 0189.RAW

Date: $\quad$ 2016-05-28 AM 11:27:06

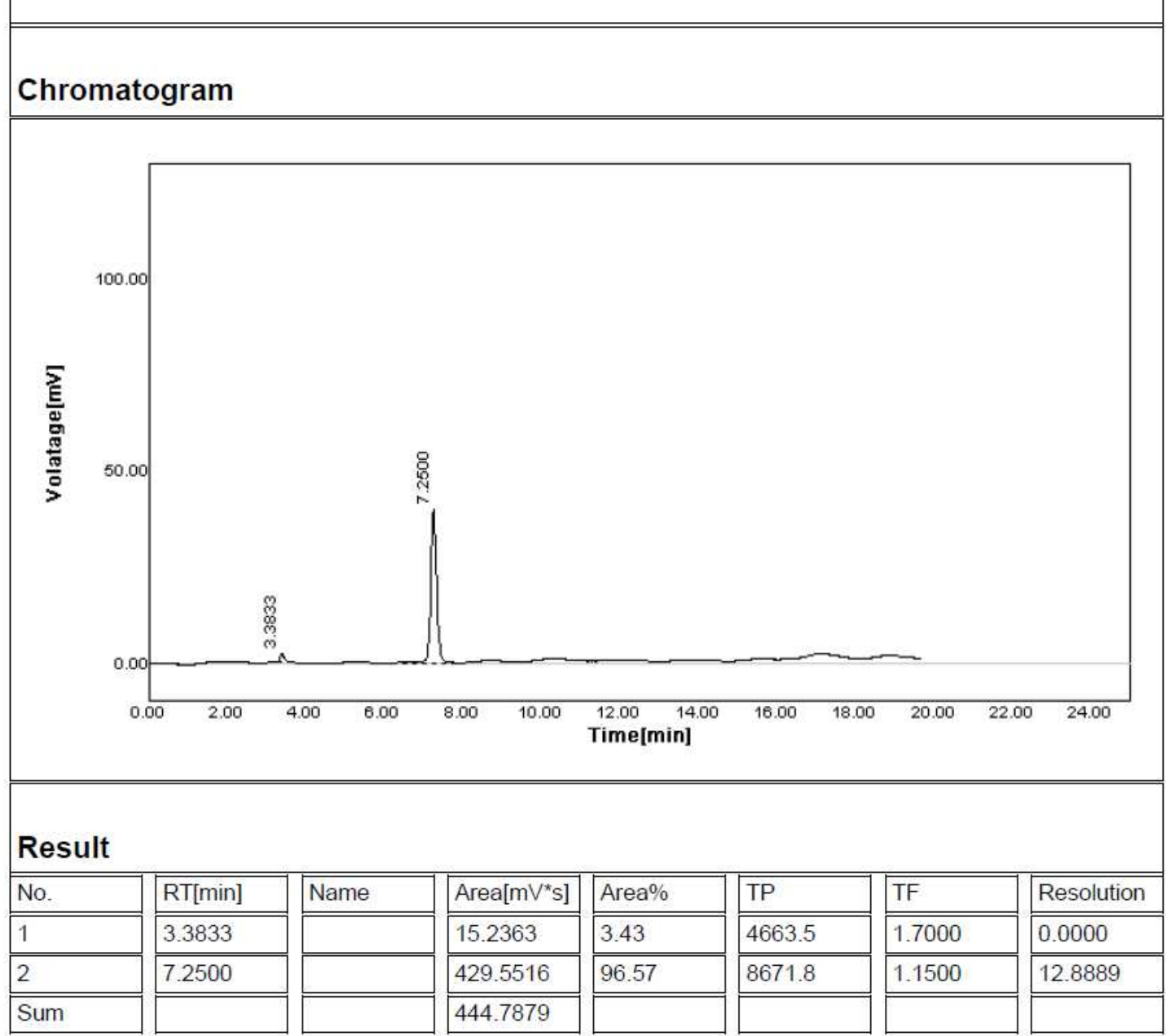

10. HPLC of TEX (Scale up) 